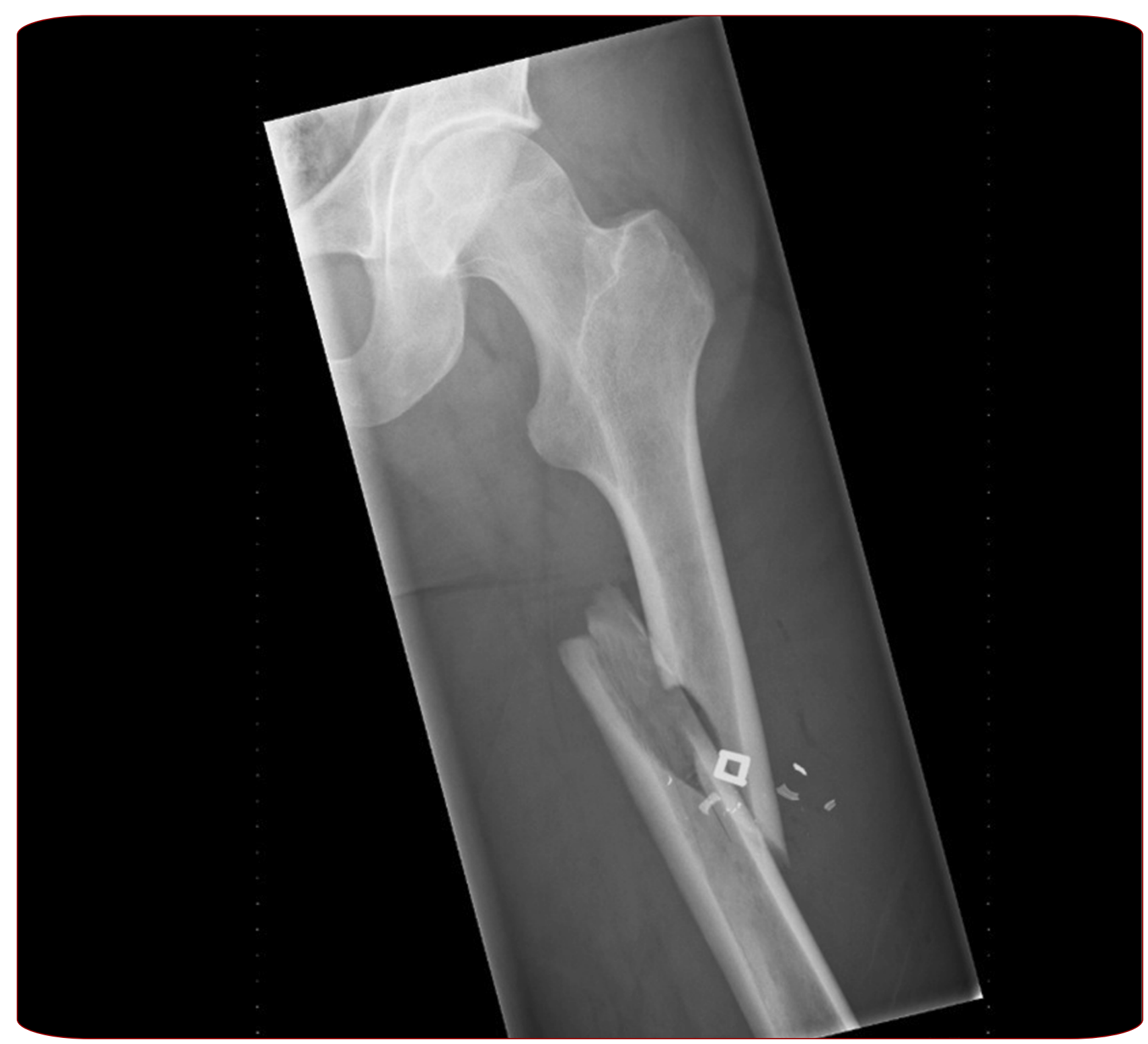

Treatment of penetrating trauma of the extremities: ten years' experience at a dutch level 1 trauma center

Waes et al. 


\title{
Treatment of penetrating trauma of the extremities: ten years' experience at a dutch level 1 trauma center
}

\author{
Oscar JF Van Waes*, Esther MM Van Lieshout, Wouter Hogendoorn, Jens A Halm and Jefrey Vermeulen
}

\begin{abstract}
Background: A selective non-operative management (SNOM) has found to be an adequate and safe strategy to assess and treat patients suffering from penetrating trauma of the extremities (PTE). With this SNOM comes a strategy in which adjuvant investigations or interventions are not routinely performed, but based on physical examination only.

Methods: All subsequent patients presented with PTE at a Dutch level I trauma center from October 2000 to June 2011 were included in this study. In-hospital and long-term outcome was analysed in the light of assessment of these patients according to the SNOM protocol.

Results: A total of 668 patients (88.2\% male; 33.8\% gunshot wounds) with PTE presented at the Emergency Department of a level 1 traumacenter, of whom 156 were admitted for surgical treatment or observation. Overall, 22 (14\%) patients that were admitted underwent exploration of the extremity for vascular injury. After conservative observation, two (1.5\%) patients needed an intervention to treat (late onset) vascular complications. Other long-term extremity related complications were loss of function or other deformity $(n=9)$ due to missed nerve injury, including 2 patients with peroneal nerve injury caused by delayed compartment syndrome treatment.

Conclusion: A SNOM protocol for initial assessment and treatment of PTE is feasible and safe. Clinical examination of the injured extremity is a reliable diagnostic 'tool' for excluding vascular injury. Repeated assessments for nerve injuries are important as these are the ones that are frequently missed and result in long-term disability. Level of evidence: II / III, retrospective prognostic observational cohort study Key words Penetrating trauma, extremity, vascular injury, complications.
\end{abstract}

\section{Background}

Penetrating trauma of the extremities (PTE) is considered a difficult injury to manage because artery and nerve injuries can be serious and may significantly impair outcome of the patient. PTE accounts for about $50 \%$ of penetrating trauma. Despite possible (long-term) complications, overall survival is very high $[1,2]$. Nevertheless, the low incidence of this kind of trauma in Western Europe makes it difficult for trauma surgeons to gain experience in its management.

A selective non-operative management (SNOM) has found to be an adequate and safe strategy to assess and treat patients suffering from PTE [3-6]. With this SNOM comes a strategy in which diagnostic computed tomography angiography (CTA) screening is not routinely

\footnotetext{
*Correspondence: o.vanwaes@erasmusmc.nl

Department of Surgery-Traumatology, Erasmus MC, University Medical Center Rotterdam, P.O. Box 2040, Rotterdam, the Netherlands
}

performed, but based on physical examination only. The accuracy of physical examination to detect vascular injury is very high in patients after penetrating trauma [3,7]. Hard signs of a vascular injury (Table 1) mandate emergent surgical exploration, or, if the patient is hemodynamically stable, endovascular treatment could be considered. Diagnostic CTA is indicated in hemodynamically stable patients with clinical signs of vascular injury (Table 1 ). Without signs of vascular impairment in PTE a conservative observational strategy without CTA is viable $[5,6,8]$.

The present study was undertaken to assess SNOM in relation to long-term outcome and complications.

\section{Patients and methods}

All patients presented with PTE at a single Dutch level I trauma center from October 2000 to June 2011 were included in this study. Data regarding age, gender, mechanism of injury, type of injury (i.e. vascular, orthopaedic,

\section{Ciomed Central}


Table 1 Signs of arterial injury [3]

\begin{tabular}{ll}
\hline Hard signs & Active hemorrhage \\
\hline & Absent distal pulses or ischemia \\
\hline Expanding or pulsatile hematoma \\
\hline Bubtle signs & Subjective reduced or unequal pulses \\
\hline & Large non-pulsatile hematoma \\
\hline $\begin{array}{l}\text { Orthopedic injuries carrying a high index } \\
\text { of suspicion of vascular injury }\end{array}$ \\
\hline Neural injury \\
\hline History of large hemorrhage on trauma scene
\end{tabular}

or nerve), anatomical location and concomitant injuries, clinical manifestations and vital parameters, indications for additional investigations, and treatment strategy of all patients were collected and analyzed in the light of patient's long-term outcome.

Appropriate approval of the local ethical review board was obtained prior to inclusion of patients into the trauma database. Due to the retrospective and anonymous nature of the database no further approval was deemed necessary by the ethical review board.

All patients were initially resuscitated according to the Advanced Trauma Life Support $\left(\right.$ ATLS $^{\circledR}$ ) [9] guidelines and to the discretion of the trauma surgeon in charge. A local protocol was established in order to manage these injuries (Figure 1): Hemodynamically stable patients, and patients who stabilize after immediate simple resuscitation, were first evaluated with a thorough history and physical examination. Additional diagnostic investigations were performed when indicated by the preset protocol based on history and clinical manifestations. A routine $\mathrm{X}$-ray of the injured extremity was made in patients with a gunshot wound (GSW). Indication for CTA was based on the presence of signs and symptoms of vascular injury found by clinical examination. Patients were immediately transferred to the operating room for surgical intervention if additional severe injuries in need of immediate surgical were diagnosed, or no preliminary hemostasis could be achieved in the ER. Hemodynamically stable patients with a negative history and clinical examination suspicious of vascular injury were admitted to the trauma surgical ward for observation. After 24 hours without complications the patient could be discharged home. All patients were instructed for alarm symptoms of vascular injury (loss of "vascular integrity" in the affected limb, e.g. expanding haematoma, loss of pulse, palor and coolness, or loss of sensation and function of the affected limb. Plus general signs of infection (erythema, swollen, warm)); if these occurred, they had to return to the hospital immediately.

Hemodynamically unstable patients were immediately transferred to the operating room. In actively bleeding

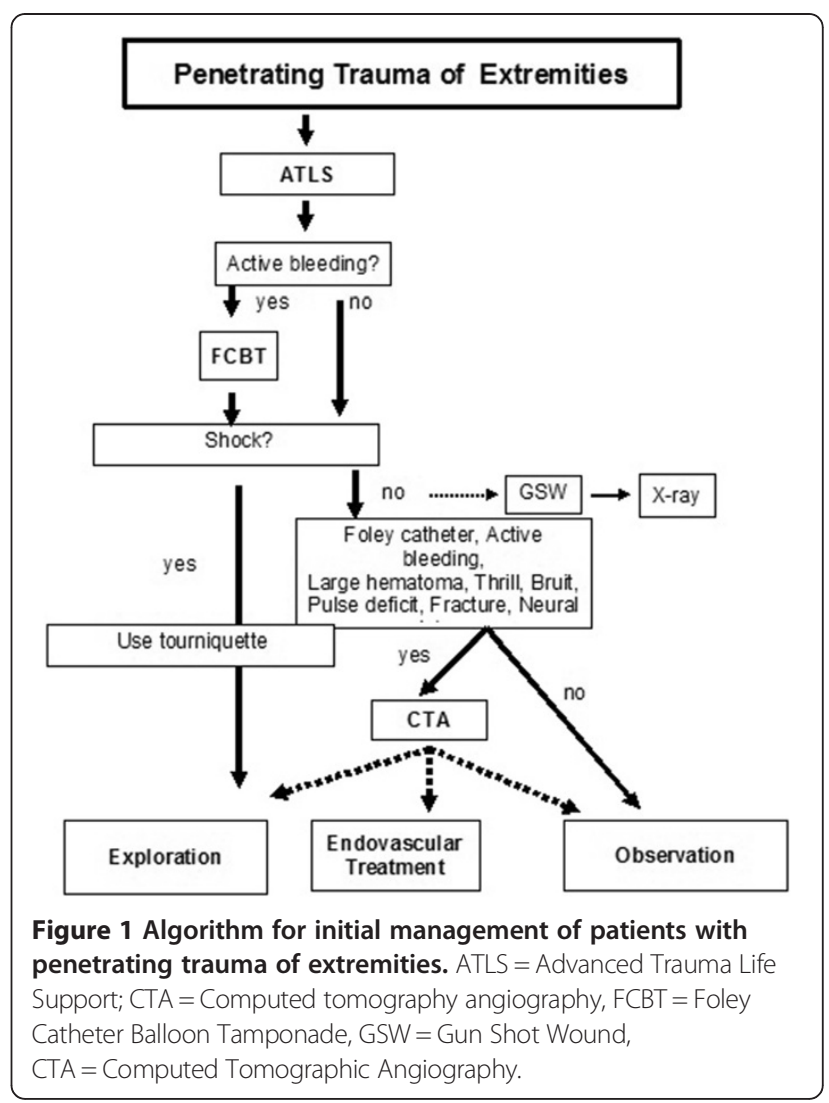

patients hemorrhage control was attempted by using a tourniquet followed by Foley catheter balloon tamponade (FCBT). If hemorrhage control was not established, surgical exploration of the injured extremity had to follow immediately. If hemorrhage was controlled by FCBT, angiography or CTA was indicated after removal of an eventual tourniquet, in order to detect major arterial injury. If positive, patients should still be transferred to the operating room or treated by endovascular stenting or coiling. Without any arterial injury deemed in need of surgical or radiological interventional (RI) treatment, the patient should be observed for 24-48 hours, after which the Foley catheter was removed in the operating room. In case of re-bleeding, surgical intervention was performed.

\section{Results}

A total of 668 patients (88.2\% male; 33.8\% GSWs) with PTE presented at the Emergency Department during the study period. After initial assessment, 512 patients were discharged home from the Emergency Department as the type and severity of their injury did not necessitate admission for observation or intervention. None of these patients returned to the hospital with late onset complications due to PTE.

Analysis of our prospective gathered trauma patient database revealed that a total of 156 patients were 
admitted after PTE. Stab wounds (SW) were found in 75 patients (10 women) and GSW in the remaining 81 patients ( 2 women). Characteristics of the patients and type and location of their sustained penetrating injuries are listed in Table 2. Sixteen patients underwent CTA as additional investigation to assess vascular integrity (Table 3). Although CTA should only be performed based on findings at physical examination with suspicion for vascular injury, according to the protocol, in four patients primary CTA was performed without relevant indication and without clinical signs of active bleeding. None of the four CTAs showed vascular injuries. Only one patient was initially treated with FCBT because of active bleeding. Subsequent diagnostic CTA showed minor arterial injury, which could be treated conservatively as no re-bleeding occurred after removal of the Foley catheter.

Twenty patients underwent emergency surgery because of ongoing bleeding or hemodynamic instability, not improving during initial resuscitation or because of extremity ischemia or specific findings at CTA. Another 20 patients underwent surgery for reasons mentioned in Table 4. Overall, 22 (14\%) patients that were admitted underwent exploration of the extremity for vascular injury. In 12 of these patients reconstruction of vascular

Table 2 Demographics of 156 patients admitted with penetrating extremity injury

\begin{tabular}{|c|c|}
\hline Sex ratio (M:F) & $144: 12$ \\
\hline Age, years (median; range) & $27(11-86)$ \\
\hline \multicolumn{2}{|l|}{ Penetrating extremity injury } \\
\hline Stab wound (female) & $75(10)$ \\
\hline Gunshot wound (female) & $81(2)$ \\
\hline \multicolumn{2}{|l|}{ Extremity injury } \\
\hline \multicolumn{2}{|l|}{ Vascular } \\
\hline Emergency exploration & 14 \\
\hline Computed tomography angiography & 8 \\
\hline \multicolumn{2}{|l|}{ Fracture } \\
\hline X-ray ${ }^{1}$ & $14(5)$ \\
\hline \multicolumn{2}{|l|}{ Neural } \\
\hline Physical examination $^{1}$ & $22(10)$ \\
\hline \multicolumn{2}{|l|}{ Concomitant penetrating injury ${ }^{2}$} \\
\hline Stab wound & 45 \\
\hline Gunshot wound & 22 \\
\hline \multicolumn{2}{|l|}{ Location } \\
\hline Head & 13 \\
\hline Neck & 12 \\
\hline Chest & 31 \\
\hline Abdomen & 29 \\
\hline Thigh/Pelvis & 3 \\
\hline
\end{tabular}

Table 3 Indications for and results of vascular investigations

\begin{tabular}{ll}
\hline Indication for investigation & CTA ( $\mathbf{n = 1 6 )}$ \\
\hline Absent or diminished pulses & $1(1)$ \\
\hline Large hematoma & $6(5)$ \\
\hline Foley catheter balloon catheter & $1(1)$ \\
\hline Bruit & $1(1)$ \\
\hline Proximity to major vessels & $3(0)$ \\
\hline Not specified & $4(0)$ \\
\hline
\end{tabular}

Values in parentheses are numbers of additional investigations with positive findings on CTA, e.g. extravasation, stop, fistula.

$\mathrm{CTA}=$ Computed tomography angiography.

injury with use of a venous graft was performed, instead of primary repair or suture ligation. No patients were treated primarily by radiological intervention. Six patients underwent surgery to repair traumatic fractures and another nine patients underwent surgery because of nerve injury. In one patient the plastic surgeon joined the trauma surgeon during fracture care surgery to repair neural injury (Table 4). Primary fasciotomy was performed in four patients: one underwent fasciotomy to treat an acute compartment syndrome, the others underwent pre-emptive fasciotomy after vascular reconstructive surgery $(n=2)$ and nerve injury repair. Fractures of the extremities after penetrating injury were almost exclusively found after GSW $(n=13)$. One metacarpal fracture was found in a patient with SW.

In 134 patients conservative observational strategy for vascular symptoms could be initialized after PTE. This equals $86 \%$ of admitted patients and $97 \%$ of all patients presented at the Emergency Department after PTE. After conservative observation, two $(1.5 \%$, or $0.3 \%$, respectively) of these patients subsequently needed an intervention to treat (late onset) vascular complications (Table 5). In one patient emergent repair of the deep femoral artery was complicated by the formation of an arterio-venous fistula discovered after clinical observation and additional CTA,

Table 4 Indications for surgical intervention

\begin{tabular}{ll}
\hline Indication for emergency exploration & $\mathbf{2 0}$ \\
\hline Active hemorrhage or shock & 9 \\
\hline Absent pulses & 5 \\
\hline Vascular injury found at CTA & 6 \\
\hline Indication for early surgery & $\mathbf{2 0}$ \\
\hline Vascular injury found at CTA & 2 \\
\hline Fracture & 5 \\
\hline Neural injury & $9^{1}$ \\
\hline Wound management & 2 \\
\hline Removal of bullet & 1 \\
\hline Fasciotomy of the lower leg & 1 \\
\hline T. One par who
\end{tabular}

1. One patient who underwent exploration because of nerve injury also was operated on to repair a metacarpal fracture.

$\mathrm{CTA}=$ Computed tomography angiography. 
Table 5 (Long-term) complications that were initially missed or had severe consequences

\begin{tabular}{|c|c|c|}
\hline Initial treatment & Complication & Consequence/result \\
\hline \multicolumn{3}{|l|}{ Stab wound } \\
\hline \multirow[t]{3}{*}{ Exploration } & $\begin{array}{l}\text {-Brain-injury due to } \\
\text { exsanguination }(n=2)\end{array}$ & Death \\
\hline & -Femoral nerve injury & Weakness leg \\
\hline & $\begin{array}{l}\text {-Arterio-venous fistula after } \\
\text { femoral a. repair }\end{array}$ & Coiling \\
\hline \multirow[t]{3}{*}{ Conservative } & -Brachial plexus lesion & Limp/ weakness arm \\
\hline & -Median nerve lesion & Ape hand deformity \\
\hline & -Ulnar nerve injury $(n=2)$ & $\begin{array}{l}\text { Paraesthesia } \\
\text { and weakness }\end{array}$ \\
\hline \multicolumn{3}{|l|}{ Gunshot wound } \\
\hline \multirow[t]{5}{*}{ Exploration } & $\begin{array}{l}\text {-Leg length difference after } \\
\text { femur fracture }\end{array}$ & Surgical correction \\
\hline & $\begin{array}{l}\text {-Sciatic nerve injury after } \\
\text { femoral a. repair }\end{array}$ & $\begin{array}{l}\text { Leg pain and } \\
\text { foot weakness }\end{array}$ \\
\hline & $\begin{array}{l}\text {-Hip joint disarticulation after } \\
\text { femoral a. injury and femur } \\
\text { fracture }\end{array}$ & Wheelchair bound \\
\hline & $\begin{array}{l}\text {-Peroneal nerve injury after } \\
\text { compartment syndrome }\end{array}$ & Foot drop \\
\hline & $\begin{array}{l}\text { compartment syndrome } \\
\text { after popliteal a. repair }(n=2)\end{array}$ & \\
\hline \multirow[t]{3}{*}{ Conservative } & -False aneurysm popliteal a. & Surgical repair \\
\hline & -Erysipelas foot due to bullet & Surgical exploration \\
\hline & -Ulnar nerve injury & Claw hand \\
\hline
\end{tabular}

which was treated by endovascular coiling. The other patient returned with a false aneurysm of the popliteal artery several months later, which was missed at CTA during first admission. This patient was successfully operated on by the vascular surgeon.

Two patients (both SW) died of diffuse axonal injury and post anoxic encephalopathy after exsanguination due to penetrating chest and extremity injury. Besides, the complications mentioned above, long-term extremity related complications were loss of function or other deformity $(n=9)$ including two patients with peroneal nerve injury caused by delayed compartment syndrome treatment, late onset infection and severe wound healing problems resulting in hip exarticulation $(\mathrm{n}=1$; combined injury of femoral artery and proximal femur).

\section{Discussion}

In the Netherlands, as in the rest of Western Europe, the incidence of penetrating injury is rather low. Due to the low incidence it is not possible for a trauma surgeon to get extensive experience with the management and treatment of this kind of trauma, causing obscurity, disagreement in diagnostic and treatment options, and an insufficient or incomplete management of this trauma patient. All together, inexperience in assessment of patients with PTE might increase the risk of mistakes and may hamper outcome.

In trauma centers that treat a higher number of patients with penetrating trauma, SNOM is becoming more and more accepted. SNOM is based on clinical examination and additional investigations (on indication). Together they have shown to be a reliable indicator of clinically significant injury, with a sensitivity and specificity of $99 \%$ and a negative predictive value of $99 \%$ $[6,10]$. The management protocol for assessing and treating patients with PTE is based essentially on hemodynamic status, together with a thorough physical examination. Adjuvant CTA is only indicated based on hard and subtle signs of vascular injury found during clinical assessment in hemodynamically stabilized patients. CTA is a reliable and accurate investigation with a sensitivity and specificity of $95 \%$ and $100 \%$ respectively, a positive predictive value of $100 \%$ and a negative predictive value of $98 \%$ [11-13]. Therefore CTA is more and more becoming the diagnostic tool of choice during initial evaluation of stable patients with suspected vascular injury, including patients after PTE $[13,14]$. The combination of FCBT and CTA could also diminish the rate of negative explorations and iatrogenic injuries. In one patient an actively bleeding groin was successfully controlled by FCBT. Subsequent CTA revealed no indication for surgical exploration, and after two days the catheter was removed without rebleeding.

In the present study the SNOM protocol for penetrating extremity injury was correctly executed with good persistence. Only four out of 124 admitted patients with no signs of vascular injury still underwent CTA. None showed signs of vascular lesions, and all four were successfully treated conservatively. Vascular observational management after PTE was applied in $86 \%$ of admitted patients without $(\mathrm{n}=126)$ or after CTA $(\mathrm{n}=8)$ assessment. During follow up only one $(0.7 \%)$ of the patients who were conservatively treated and observed returned with symptoms of a false aneurysm several months later. This indicates that initial conservative management (or SNOM) of patients with PTE is feasible and safe.

Although the majority of patients presented at the Emergency Department with supposed PTE are not seriously injured and can be discharged after physical examination and treatment of wounds, up to a quarter of patients should be admitted for observation, additional investigations or surgical treatment. The total surgical treatment rate of the latter group was $24 \%$ (22 vascular injuries, five fractures, 10 exclusively neural injuries), indicating that PTE should be considered a serious trauma which requires intensive and thorough assessment of the extremities. PTE is frequently accompanied by other penetrating injuries (in this study in $43 \%$ of cases), that possibly needs to be managed first or 
distracts the physician's attention away from the injuries of the extremities. Eventually missed or even delayed assessment of PTE may significantly impair outcome of the patient $[15,16]$.

In the present study, seven patients (5\%) who were treated conservatively showed symptoms of nerve injury that were missed during the initial hospital stay. Although the larger part of nerve injuries cannot be treated, it is important to recognize these injuries at initial assessment, in order to adequately inform patients and provide supportive treatment. These are important factors in the rehabilitation process after penetrating trauma, especially for patients with prolonged or definitive impairment of the extremity [17].

Not only is it important to recognize nerve injury at initial assessment, it is of vital importance to prevent nerve injury in a later stage of treatment. Of all 12 patients that underwent primary vascular repair, only two underwent fasciotomy during the same vascular-reconstructive operation in order to prevent compartment syndrome. In two (20\%) patients who had not undergone fasciotomy, compartment syndrome after revascularisation of the leg was diagnosed too late, resulting in persistent peroneal nerve injury. In other words, a patient sustaining PTE should not only be intensively reassessed several times during conservative treatment, but also after surgical treatment, not only for vascular injury, but nerve injury as well. Besides, preemptive fasciotomy is advised, in patients sustaining a combination of arterial and venous injury, multiple or complex fractures and an ischemia time longer than six hours $[18,19]$, as continuous compartment pressure-monitoring is not reliable. Blood flow should be restored as soon as possible by using a shunt. After initial shunting, fractures should be rigidly stabilized using external fixation devices, in order to perform definitive vascular repair with a tension free (venous) interposition graft [20]. Since these repairs usually take a fair amount of time, there is a serious threat of compartment syndrome after revascularisation. Therefore, a pre-emptive fasciotomy is highly recommended.

In summary, the low failure rate in this study validates the SNOM protocol for initial management of PTE. Clinical examination of the injured extremity is a reliable diagnostic approach for excluding vascular injury. It is important to assess for possible nerve injuries, both preand post operatively, as these injuries are frequently missed and might result in long-term disability.

\section{Competing interests}

The authors declare that they have no competing interests.

\section{Authors' contribution}

OW: Writing of the article, data interpretation, approved final version, EML: Study design, data interpretation, critical revision, approved final version, WH: Data collection, approved final version, JAH: Data collection, literature search, approved final version, JV: Study design, data analysis, data interpretation, writing, approved final version.
Received: 14 September 2012 Accepted: 3 January 2013

Published: 14 January 2013

\section{References}

1. Doody O, Given MF, Lyon SM: Extremities-indications and techniques for treatment of extremity vascular injuries. Injury 2008, 39:1295-303.

2. Manthey DE, Nicks BA: Penetrating trauma to the extremity. J Emerg Med 2008, 34:187-93.

3. Dennis JW, Frykberg ER, Veldenz HC, Huffman S, Menawat SS: Validation of nonoperative management of occult vascular injuries and accuracy of physical examination alone in penetrating extremity trauma: 5- to 10-year follow-up. J Trauma 1998, 44:243-52.

4. Dragas M, Davidovic L, Kostic D, Markovic M, Pejkic S, Ille T, llic N, Koncar I: Upper extremity arterial injuries: factors influencing treatment outcome. Injury 2009, 40:815-9.

5. Stone WM, Fowl RJ, Money SR: Upper extremity trauma: current trends in management. J Cardiovasc Surg 2007, 48:551-5.

6. Van Waes OJF, Navsaria PH, Verschuren RCM, Vroon LC, Van Lieshout EMM, Halm JA, Nicol AJ, Vermeulen J, Frykberg ER, Dennis JW, Bishop K, Laneve L, Alexander RH: Management of penetrating injuries of the upper extremities. (submitted)7. The reliability of physical examination in the evaluation of penetrating extremity trauma for vascular injury: results at one year. J Trauma 1991, 31:502-511.

7. Keen JD, Dunne PM, Keen RR, Langer BG: Proximity arteriography: cost effectiveness in asymptomatic penetrating extremity trauma. J Vasc Interv Radiol 2001, 12:813-2.

8. Corballis B, Nitowski L: Prim Care. Advanced trauma life support 1986, 13:33-44.

9. Peng PD, Spain DA, Tataria M, Hellinger JC, Rubin GD, Brundage SI: CT angiography effectively evaluates extremity vascular trauma. Am Surg 2008, 74:103-7.

10. Wallin D, Yaghoubian A, Rosing D, Walot I, Chauvapun J, de Virgilio C: Computed tomographic angiography as the primary diagnostic modality in penetrating lower extremity vascular injuries: a level I trauma experience. Ann Vasc Surg 2011, 25:620-3.

11. Soto JA, Múnera F, Cardoso N, Guarín O, Medina S: Diagnostic performance of helical $C T$ angiography in trauma to large arteries of the extremities. J Comput Assist Tomogr 1999, 23:188-96.

12. Seamon MJ, Smoger D, Torres DM, Pathak AS, Gaughan JP, Santora TA, Cohen $\mathrm{G}$, Goldberg AJ: A prospective validation of a current practice: the detection of extremity vascular injury with CT angiography. $J$ Trauma 2009, 67:238-43.

13. Halvorson JJ, Anz A, Langfitt M, Deonanan JK, Scott A, Teasdall RD, Carroll EA: Vascular injury associated with extremity trauma: initial diagnosis and management. J Am Acad Orthop Surg 2011, 19:495-504.

14. Hafez HM, Woolgar J, Robbs JV: Lower extremity arterial injury: results of 550 cases and review of risk factors associated with limb loss. J Vasc Surg 2001, 33:1212-9.

15. Richardson JD, Vitale GC, Flint LM Jr: Penetrating arterial trauma. Analysis of missed vascular injuries. Arch Surg 1987, 122:678-83.

16. Jaquet JB, Kalmijn S, Kuypers PD, Hofman A, Passchier J, Hovius SE: Early psychological stress after forearm nerve injuries: a predictor for long-term functional outcome and return to productivity. Ann Plast Surg 2002, 49:82-90.

17. Farber A, Tan TW, Hamburg NM, Kalish JA, Joglar F, Onigman T, Rybin D, Doros $G$, Eberhardt RT: Early fasciotomy in patients with extremity vascular injury is associated with decreased risk of adverse limb outcomes: A review of the National Trauma Data Bank. Injury 2011, 28. Epub ahead of print.

18. Zaraca F, Ponzoni A, Stringari C, Ebner JA, Giovannetti R, Ebner H: Lower extremity traumatic vascular injury at a level II trauma center: an analysis of limb loss risk factors and outcomes. Minerva Chir 2011, 66:397-407.

19. Desai P, Audige L, Suk M: Combined orthopedic and vascular lower extremity injuries: sequence of care and outcomes. Am J Orthop (Belle Mead NJ) 2012, 41:182-6.

20. Gifford SM, Eliason JL, Clouse WD, Spencer JR, Burkhardt GE, Propper BW, Dixon PS, Zarzabal LA, Gelfond JA, Rasmussen TE: Early versus delayed restoration of flow with temporary vascular shunt reduces circulating markers of injury in a porcine model. J Trauma 2009, 67:259-65.

doi:10.1186/1757-7241-21-2

Cite this article as: Waes et al.: Treatment of penetrating trauma of the extremities: ten years' experience at a dutch level 1 trauma center. Scandinavian Journal of Trauma, Resuscitation and Emergency Medicine 2013 21:2. 